\title{
Older drivers, medical condition, medical impairment and crash risk
}

\author{
F. Javier Alvarez*, Inmaculada Fierro \\ Faculty of Medicine, University of Valladolid, 47005 Valladolid, Spain \\ Received 9 August 2006; received in revised form 2 March 2007; accepted 8 April 2007
}

\begin{abstract}
New evidence has appeared to support the fact that the over-involvement of older drivers in traffic accidents disappears when the low mileage bias is taken into account. As a group, older drivers are as safe as or safer than other age groups, and only low mileage older drivers have a high crash rate. Furthermore, the role of the medical condition of older drivers in traffic accidents, as well as the fitness to drive evaluation, are objects of controversy. We examined all this with a cohort of 4316 drivers attending Medical Driving Test Centres for a mandatory fitness to drive evaluation. Our data shows that older drivers $(\geq 75)$ have a lower crash rate. Medical conditions that impair fitness to drive, as a tendency, increased with advanced age and with lower mileage group. The multivariate analysis of variance showed that there is an effect $(p<0.0001)$ of age-range and mileage on the annual crash rate per million kilometres driven, while a medical restriction ("fit to drive with restriction") has no effect ( $p>0.05$ ). Our data suggests that health status is not associated with increased crash risk for the low mileage group, although further studies are needed. (C) 2007 Elsevier Ltd. All rights reserved.
\end{abstract}

Keywords: Older drivers; Low mileage bias; Crash risk; Health status

\section{Introduction}

In developed countries, an increasing proportion of the population is aged, and many of these aged people drive a motor vehicle. Despite the availability of public transport facilities, possessing a driver's licence and driving a motor vehicle may imply a higher degree of mobility and freedom. Concern about driving by aged people is an increasing issue for both the population and road traffic administrations. Even the question of when a person is "aged" or feels aged (65?, 75 years old?), is a sensitive issue. According to the OECD report on Ageing and Transport (2001), by 2030, 1 out of 4 drivers will be aged 65 and over. The report also claims that the most critical safety issue for older people (either drivers or passengers) relates to their increased frailty and associated increase in injury susceptibility. Without active intervention, it has been estimated that over the next three decades fatal crashes could be as much as three times higher among older drivers (Hu et al., 2000).

In general, several studies have shown that older drivers have increased crash rates. This is more evident when an exposure measure (yearly distance driven, for example) is taken into account for calculating risk (Li et al., 2003; Langford et al.,

\footnotetext{
* Corresponding author. Tel.: +34 983423077; fax: +34 983423022 .

E-mail address: alvarez@med.uva.es (F.J. Alvarez).
}

2006). Due to greater vulnerability to injury (reductions in bone strength and fracture tolerance), these figures must be corrected by the so-called "frailty bias" (Li et al., 2003; Langford et al., 2006). Between 60 and $95 \%$ of the increase in death rate per distance travelled for those aged 60 and over can be accounted for by increases in fragility (Li et al., 2003).

This approach has recently been questioned, and a new element has been proposed for analysis (Hakamies-Blomqvist et al., 2002; Langford et al., 2006): the so-called low mileage bias (Langford et al., 2006). This approach indicates that, independent of age, drivers travelling will usually have lower crash rates per kilometre than those driving fewer kilometres. Older drivers tend to drive less distance per trip and hence have a lower accumulated driving distance per year. They, therefore, have greater crash involvement per unit of distance as compared to drivers with greater accumulated driving distances.

Langford et al. (2006) used Dutch data from 47,502 drivers to confirm previous research findings (HakamiesBlomqvist et al., 2002) concerning the association between annual mileage driven and crash involvement, as well as the influence of the so-called low mileage bias. Crash rates per million driver-kilometres for low mileage ( $<3000 \mathrm{~km}, n=8035)$, mid mileage (3000-14,000 km, $n=19,704)$ and high mileage $(>14,000 \mathrm{~km}, n=19,763)$ drivers were calculated for different age-ranges (18-20, 21-30, 31-64, 65-74 and +75 years of age). It was observed that, as annual mileage increased, the crash rate 
per distance travelled declined; those who drove $<3000 \mathrm{~km} / \mathrm{year}$ had a crash rate six times that of those who drove $\geq 14,000 \mathrm{~km}$. Furthermore, the older the driver, the lower the crash involvement, except for drivers who drove $<3000 \mathrm{~km} / \mathrm{year}$, where the decline in crash involvement as age increased is reversed for the oldest drivers ( +75 years of age). Furthermore, low mileage drivers of all age-ranges had a significantly higher crash rate than drivers of the same age-range with medium mileages. The same applies for medium mileage drivers compared to high mileage drivers. Langford et al. (2006) highlight that most drivers aged 75 years and above were safer than younger age groups (after crash rates by age-range were matched for yearly driving distance). "Only low mileage older drivers (just over $10 \%$ of older drivers in the survey) indicated a relatively high self-reported crash rate. As a group, older drivers were as safe as or safer than other age groups" (Langford et al., 2006, p.p. 576).

Furthermore, they conclude (Langford et al., 2006, p.p. 577): "However, since the findings clearly support the overall conclusion that older drivers are not a high-risk group, a major conclusion follows. There are no rational grounds for implementing mandatory age-based testing of driving fitness for a group, the large majority of whose members are demonstrably as safe as or safer than drivers of other ages. Attempts to identify high-risk drivers should focus upon those drivers giving some preliminary evidence of being at-risk, without involving all older drivers in a formal assessment process".

There are several issues not explored in the study of these drivers that could affect the road crash rate. As the fitness to drive evaluation deals with the medical condition of the driver, one issue that should be assessed prior to proposing this conclusion is the medical/ophthalmologic condition of the driver. For example, is the medical condition of the low mileage drivers group different with regard to the medium and high mileage groups?

Current European legislation (The Council of the European Communities, 1991) does not permit the issuing or renewal of driving licences for those who do not possess adequate driving ability. Council Directive 91/439/CEE on driving licences establishes, in Annex III, the minimum standards of physical and mental fitness for driving a power-driven vehicle. In practice, various types of illness and disease, including the effects of medical treatment, can affect fitness to drive.

Member states implement this regulation differently (European Union and European Economic Area, 2006). For example, Spanish legislation establishes that to obtain a driving licence or to renew it (every 10 years up to 45 , every 5 years between 46 and 70 and every 2 years from 70 onwards for non-professional drivers, and every 5 years until 45, every 3 years until 60 and evey 2 years from 60 onwards for professional drivers), a medical-psychological examination carried out in specific 'Medical Driving Test Centres', is obligatory. In these Medical Driving Test Centres, medical, eyesight and psychological tests are carried out with a view to assessing fitness to drive in accordance with Spanish legislation (Royal Decree 1598/2004).

For many years, Spain has been the only European Union country where a mandatory fitness to drive examination is per- formed on all drivers at regular time intervals. We should point out that a study in Spain on whether or not the system (mandatory fitness to drive evaluation) is cost-effective has never been carried out, nor has the impact on road-safety been evaluated. Furthermore, no national data-base exists regarding the results of the approximately 2 million annual fitness to drive examinations. We have, therefore, used the data gathered within the framework of the EU project IMMORTAL (Alvarez et al., 2004).

The aim of the study was to analyze road traffic accident rates by age-range, taking into account the low mileage bias, as well as several medical issues of the drivers. Based on the findings reported by Langford et al. (2006), we expected older drivers' over-involvement in traffic accidents to disappear when the low mileage bias was taken into account. We also expected a medical restriction (the driver being rated as "fit to drive with restrictions") to be related to low mileage older drivers.

\section{Material and methods}

\subsection{Fitness to drive assessment}

Fitness to drive in Spain is evaluated in Medical Driving Test Centres, following Spanish regulations (Royal Decree 1598/2004) and EU rules (CD 91/439/CEE), by an ophthalmologist, a psychologist and a general practitioner. Each one examines the driver in particular areas fitting their specialization. The medical issues examined are: (i) visual capacity, (ii) auditive capacity, (iii) locomotor system, (iv) cardiovascular system, (v) hematological disorders, (vi) renal system, (vii) respiratory system, (viii) metabolic and endochrinous illnesses, (ix) central nervous system, (x) mental and behavioural disorders, (xi) substance-use related disorders (alcohol and drug dependence), (xii) perceptive-motor aptitude and (xiii) any other disorder not mentioned that can impair driving ability.

Finally, the three professionals reach a decision concerning fitness to drive. The purpose is to check that the driver complies with the minimum requisites to drive safely. The stringency of the requisites will depend on the type of licence requested (professional or not).

Drivers who do not comply with the necessary conditions to drive safely are declared "unfit"; those who can drive, but with certain restrictions (e.g., changes to the vehicle, speed limits or reduced periods of licence validity) are declared "fit to drive with restrictions"; those found to comply with the necessary conditions are declared "fit".

\subsection{Participants}

A prospective study was designed that included drivers that attended two Medical Driving Test Centres in order to obtain or renew a driving licence. All drivers participating in the study were followed for 1 year to evaluate their involvement in traffic accidents. The overall results from the study have been published elsewhere (Alvarez et al., 2004) and can be seen at the IMMORTAL home web page (http://www.immortal.or.at). The study was approved by the Clinical Research Ethics Committee at the Valladolid Faculty of Medicine. 
The original study included 5234 drivers. However, in the present study, only 4316 were considered. Nine hundred and eighteen drivers were not considered because the medical examination was related to obtaining a driving licence and, therefore, they had not driven in the previous year (Alvarez et al., 2004).

Age-ranges were as follows: $<30$ years of age, $31-64,65-74$ and 75 or more years of age. In the Langford et al. (2006) study, the two lower age groups were $<20$ and 21-30 years of age. We have merged these two groups in one single group due to the limited number of drivers $<20$ years $(n=25)$ undergoing fitness to drive evaluation who had driven during the previous 12 months.

\subsection{Measures}

Crash rates per millon kilometres driven were calculated from the previous 12 months, as well as for the 12 months following the fitness to drive assessment. This information was derived from drivers' self-reported information. In this case, we have only included crash accidents as a motor vehicle driver. Any crash of any severity was considered. As in the Langford et al. (2006) study, crash rates per million driver-kilometres for low mileage $(<3000 \mathrm{~km}, n=650)$, medium mileage (3000-14,000 km, $n=1805)$, and high mileage $(>14,000 \mathrm{~km}, n=1860)$ drivers were estimated.

For the present study, we used the following clinical data from drivers with a driving licence who had driven in the previous 12 months: (i) presence of any illness in the previous year lasting over 7 days and (ii) presence of an illness that impairs driving ability, according to EU and Spanish regulations, and, therefore, deems the driver "fit to drive with restrictions" or "unfit".

\subsection{Analysis}

The SPSS program, version 13, was used for the statistical analysis of the data. Annual (year before and year after fitness to drive assessment) crash rates per million driver-kilometres (mean, 95\% confidence interval $(95 \% \mathrm{CI})$ ), prevalence of suffering from any illness in the previous year lasting over 7 days, and prevalence of being rated "fit to drive with restrictions" for the presence of an illness that impairs driving ability, between age-ranges and by mileage category, were tested for statistical significance by means of the Chi-square test (categorical variables) and analyses of variance (continuous and discrete variables). We have conducted multivariate analysis of variance (MANOVA) for repeated measures with age-range and mileage as the independent variables, with the presence of a medical restriction ("fit to drive with restriction") as a fixed factor, and annual (year before and year after fitness to drive assessment) crash rate per million kilometres driven as repeated measure. $p$-Value of $\leq 0.05$ was considered statistically significant.

\section{Results}

Overall, the annual crash rate (Table 1) was 7.4/million driver-kilometres, noticeably higher among low mileage drivers (11.8) than medium (9.3) and high mileage drivers (4.0,
$F=12.481, p<0.0001)$. The lower the mileage, the higher the likelihood of suffering an illness and being rated as "fit to drive with restrictions" (Table 1).

Table 1 shows annual crash rates per million driver-kilometres for the different driver age-ranges for the year before fitness to drive assessment: 14.6 for those aged $\leq 30$ years of age, 5.9 for those aged 31-64, 7.3 for those aged $65-74$ and 6.2 for those aged 75 or over $(F=8.796, p<0.0001)$. Within each kilometre-driver group, differences were found regarding those aged $\leq 30$ years of age $(p<0.0001)$ and those aged 31-64 $(p<0.001)$, but not for those aged $65-74$ and 75 or over. For each age-range and for the total, high mileage drivers showed a lower crash rate than medium mileage drivers (Table 1). However, medium mileage drivers did not show a lower crash rate than low mileage drivers (Table 1; for a given age-range, upper and lower $95 \%$ confidence intervals for medium and low mileage drivers are not outsiders).

Table 1 also shows the number of drivers that reported any illness, chronic or acute (lasting over 7 days) in the past year. The older the age group, the more frequent the reporting of an illness, ranging from $27.7 \%$ among the younger drivers to $78.3 \%$ for those aged 75 or more $\left(X_{3}^{2}=281.156, p<0.0001\right)$. For all the age-ranges, the following trend emerged: the lower the number of kilometres driven, the higher the frequency of reporting a past year illness. Significant differences were found only for the two younger groups.

Figures for those drivers who had an illness that potentially impairs driving performance and, after medical/ophthalmologic examination were rated "fit to drive with restrictions" due to their medical condition were 16.2 and $16.1 \%$ for those aged $\leq 30$ years of age and aged 31-64, respectively. This figure increased markedly to $30.6 \%$ for those aged $65-74$ years of age and $40.6 \%$ for those aged 75 or over (Table $1, X_{3}^{2}=119.311, p<0.0001$ ). For all the age-ranges, the lower the number of kilometres driven, the higher the frequency of having an illness that could impair the ability to drive, although differences were only statistically significant for the two younger age groups (Table 1).

If we look at the age-range group of drivers aged 75 or more, 39 out of the 85 drivers $(46.4 \%)$ that drove $\leq 3000 \mathrm{~km} /$ year had an illness that potentially impairs driving performance and were rated as "fit to drive with restrictions". This figure was 37.2\% (32 out of 86) for those who drove $3001-14,000 \mathrm{~km} / \mathrm{year}$ and $20.0 \%$ ( 2 out of 10 ) for those who drove $+14,000 \mathrm{~km} /$ year. As pointed out previously, the differences were not statistically significant ( $p>0.05$, there is a limited number of drivers involved in the study in this age-range). It should be noted that of the 5 drivers aged 75 or more who had been involved in a road traffic accident (Table 1), 2 (both in the medium mileage group) were rated as "fit to drive with restrictions", and on an annual basis had to pass a medical review prior to renewing their driving licence. The other three cases were drivers rated as fit to drive.

Finally, Table 1 shows annual crash rates per million driver-kilometres for the year after fitness to drive assessment. The reported figures were lower than those of the previous year, but only statistically significant for those aged $<30$ years of age $(t=2.583, p<0.01)$, and for the total population (from 7.41 to $5.32, t=2.618, p<0.01)$. 
Table 1

Annual crash involvement and fitness to drive evaluation for age-range and annual mileage driven

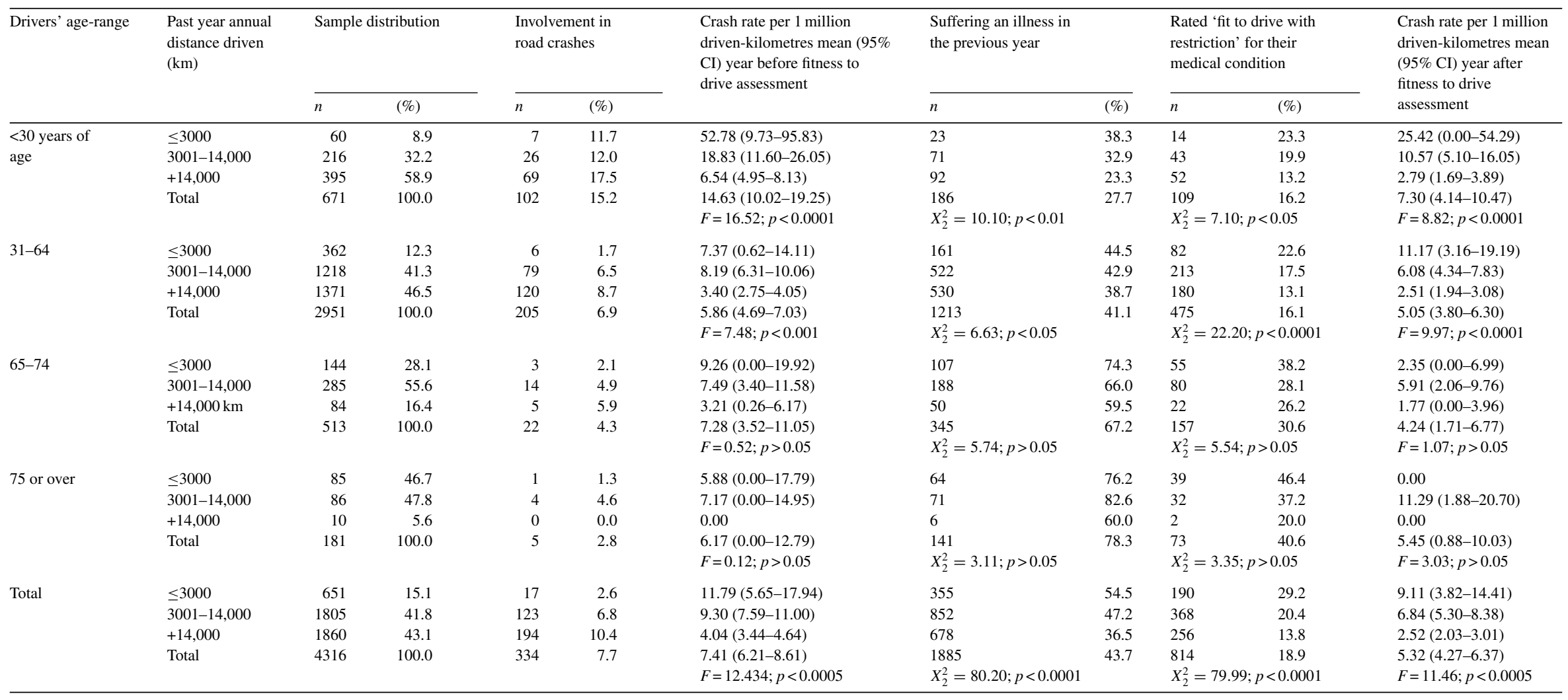


Table 2

Fitness to drive evaluation and annual crash involvement for annual mileage driven

\begin{tabular}{|c|c|c|c|c|c|}
\hline & \multirow[t]{2}{*}{$\begin{array}{l}\text { Annual distance } \\
\text { driven }(\mathrm{km})\end{array}$} & \multicolumn{2}{|c|}{$\begin{array}{l}\text { Year before fitness to drive } \\
\text { assessment }\end{array}$} & \multicolumn{2}{|c|}{$\begin{array}{l}\text { Year after fitness to drive } \\
\text { assessment }\end{array}$} \\
\hline & & $n$ & $\begin{array}{l}\text { Crash rate per } 1 \text { million } \\
\text { driven-kilometres mean } \\
(95 \% \mathrm{CI})\end{array}$ & $n$ & $\begin{array}{l}\text { Crash rate per } 1 \text { million } \\
\text { driven-kilometres mean } \\
(95 \% \mathrm{CI})\end{array}$ \\
\hline Fit & $\begin{array}{l}\leq 3000 \\
3001-14,000 \\
+14,000 \\
\text { Total }\end{array}$ & $\begin{array}{r}460 \\
1437 \\
1604 \\
3501\end{array}$ & $\begin{array}{l}11.96(4.95-18.96) \\
9.99(8.01-11.96) \\
3.78(3.16-4.40) \\
7.40(6.14-8.66) \\
F=13.97 ; p<0.001\end{array}$ & $\begin{array}{r}454 \\
1424 \\
1587 \\
3465\end{array}$ & $\begin{array}{l}9.91(3.24-16.59) \\
7.30(5.50-9.11) \\
2.44(1.93-2.95) \\
5.42(4.25-6.59) \\
F=11.51 ; p<0.0001\end{array}$ \\
\hline Fit to drive with restrictions & $\begin{array}{l}\leq 3000 \\
3001-14,000 \\
+14,000 \\
\text { Total }\end{array}$ & $\begin{array}{l}181 \\
361 \\
247 \\
789\end{array}$ & $\begin{array}{l}11.97(0.00-25.12) \\
6.75(3.42-10.08) \\
5.44(3.38-7.50) \\
7.54(4.11-10.96) \\
F=1.01 ; p>0.05\end{array}$ & $\begin{array}{l}177 \\
358 \\
241 \\
776\end{array}$ & $\begin{array}{l}7.53(0.00-16.20) \\
5.11(2.30-7.92) \\
3.14(1.55-4.72) \\
5.05(2.65-7.45) \\
F=0.85 ; p>0.05\end{array}$ \\
\hline Unfit & $\begin{array}{l}\leq 3000 \\
3001-14,000 \\
+14,000 \\
\text { Total }\end{array}$ & $\begin{array}{r}9 \\
7 \\
9 \\
25\end{array}$ & $\begin{array}{l}, 00 \\
, 00 \\
11.54(0.00-26.66) \\
4.16(0.00-9.39) \\
F=2.73 ; p>0.05\end{array}$ & & \\
\hline
\end{tabular}

The multivariate analysis of variance for repeated measures (annual crash rates per million driver-kilometres) showed that there is an effect of age-range $(F=26.463, p<0.0001)$ and mileage $(F=64.876, p<0.0001)$ on the annual crash rate per million kilometres driven, while a medical restriction ("fit to drive with restriction") has no effect $(F=0.253, p>0.05)$.

Table 2 shows annual crash rates per million driver-kilometres regarding whether or not the driver was "fit", "fit to drive with restrictions" or "unfit". Among those "fit" drivers, there are differences regarding mileage on the annual crash rate per million driver-kilometres, either for the year before $(p<0.001)$ or the year after $(p<0.001)$, being higher among the high mileage group. As a tendency, this applies to the "fit to drive with restrictions" drivers, although differences were not statistically significant (in both cases, $p>0.05)$. The "unfit" drivers involved in road traffic accidents were all included in the high mileage group (obviously they stopped driving and were not followed for the following year).

\section{Discussion}

The present study shows that the annual crash rate per million kilometres driven varies according to (i) annual mileage, with high mileage drivers being those with a lower rate and ii) agerange, with the youngest ( $<30$ years of age) being those with the higher rate. For each age-range, high mileage drivers showed a lower crash rate than medium and low mileage drivers. As a trend, for all the age-ranges, the lower the number of kilometres driven, the higher the frequency of reporting a past year illness as well as having an illness that could impair the ability to drive. Finally, the multivariate analysis of variance shows that age and mileage contributed to the outcome as measured by the annual crash rate per million kilometres driven, while whether or not the drivers had a medical restriction ("fit to drive with restriction") did not. Our study supports the previous findings by Langford et al. (2006) that older drivers' over-involvement in traffic accidents disappears when the low mileage bias is taken into account.

Langford et al. (2006) have disagreed with the need for mandatory fitness to drive evaluations and propose an assessment for high-risk drivers. In theory this is justifiable, but in practice it is difficult to understand how high-risk older drivers are identified and managed. In fact, the Spanish system of mandatory fitness to drive evaluation uses the screening approach. The most frequent restriction is the reduction of the validity of the licensing period; that is, the patients need to go every 1,2, 3 or more years for a new evaluation. Our data show that having a medical restriction ("fit to drive with restrictions") is not associated with increased crash risk for the low mileage group (directly opposing our initial hypothesis), supporting the view proposed by Langford et al. (2006) of assessment for highrisk drivers.

Langford and Koppel (2006) have reviewed the issues for and against mandatory age-based assessment of older drivers. In favour, there is the age-related decline in sensory, physical and cognitive areas related to driving, as well as the underlying medical conditions, with substantial individual differences existing. Against it, (i) it lacks effectiveness (Hakamies-Blomqvist et al., 1996; Grabowski et al., 2004; Morrisey and Grabowski, 2005), (ii) it prompts premature cessation of driving, (iii) it prompts the use of less safe alternative transport modes (e.g., increase in walking to maintain mobility) and (iv) it induces the loss of the individual benefits of maintaining mobility. They conclude that there are many more aspects against than in favour of mandatory age-based assessment of older drivers. They suggest a "strategic approach, relying upon referral only of identified atrisk drivers for multi-tiered assessment" (Langford and Koppel, 2006; p.p. 361). It should be pointed out that only recent studies 
have shown benefits for fitness to drive evaluation (Levy et al., 1995; Marshall et al., 2002).

The present study has some limitations. First, the data belongs to a "clinical" sample (drivers submitted to a fitness to drive evaluation), and, therefore, could not be generalized to the general population of drivers. Second, crash involvement was derived from drivers' self-reported information. Third, the sub-sample size in some categories was small (e.g., the 75 or over age group accounts for 181 subjects, 10 subjects in the high mileage age group). Finally, we have considered in this study any kind of medical condition that can impair fitness to drive according to the in force legislation (Royal Decree 1598/2004). Future studies should perhaps focus on medical impairments related to mental functioning, which are expected to have a greater effect on crash rates than those medical impairments not affecting mental functioning ("the more cognitive capacity approaches its limits, the greater the increase in rate of accidents", Elvik, 2006; p.p. 743)).

The Langford et al. (2006) study presents relevant data to better understand the trends in accident rates by aged drivers, especially the relevance of the low mileage bias, which is supported by the results of the present study. What characterizes those older drivers who drove less distance and had a high crash risk is not well known The present data show that having a medical restriction ("fit to drive with restrictions") has no influence on annual crash rate per million driver-kilometres. Much more research is needed in this field. We agree with Levy et al. (1995), who indicated that driving licence renewal requirements represent one of the few public policies with the potential to have a direct effect on senior traffic safety. Decisions about safe 'enough' are decisions about how much risk the society is willing to tolerate" and "decisions about the level of acceptance risk are not scientific but political" (Hakamies-Blomqvist (2006, p.p. 351)).

\section{Acknowledgements}

This study was carried out as part of the project of the European Union IMMORTAL "Impaired Motorist, Methods of Roadside Testing and Assessment for Licensing", contract no. GMA1/2000/27043 SI2.319837, program "Competitive and Sustainable Growth".

\section{References}

Alvarez, F.J., Del Río, M.C., Fierro, I., Vicondoa, A., Ozcoidi, M., 2004. Medical condition and fitness to drive: prospective analysis of the medical-psychological assessment of fitness to drive and accident risk. Deliverable R1.4 of EU-project IMMORTAL. INEAD, Valladolid, http://www.immortal.or.at/deliverables.php.

Elvik, R., 2006. Laws of accident causation. Accid. Anal. Prev. 38 (4), 742 747.

European Union and European Economic Area, 2006. Driving licenses. European Commission, Directorate General for Energy and Transport, Brussels.

Grabowski, D.C., Campbell, C., Morrisey, M.A., 2004. Elderly licensure laws and motor vehicle fatalities. JAMA 291 (23), 2840-2846.

Hakamies-Blomqvist, L., Johansson, K., Lundberg, C., 1996. Medical screening of older drivers as a traffic safety measure- a comparative Finnish-Swedish evaluation study. J. Am. Geriatr. Soc. 44 (6), 650-653.

Hakamies-Blomqvist, L., Raitanen, T., O’Neill, D., 2002. Driver ageing does not cause higher accident rates per km. Transport. Res., Part F 5 (4), 271-274.

Hakamies-Blomqvist, L., 2006. Are there safe and unsafe drivers? Transport. Res., Part F 9 (5), 347-352.

Hu, P., Jones, D., Reuscher, T., Schmoyer, R., Truett, T., 2000. Projecting fatalities in crashes involving older drivers. Report for the National Highway Traffic Safety Administration. Oak Ridge National Laboratory, ONRL, Tennessee.

Langford, J., Methorst, R., Hakamies-Blomqvist, L., 2006. Older drivers do not have a high crash risk-a replication of low mileage bias. Accid. Anal. Prev. 38 (3), 574-578

Langford, J., Koppel, S., 2006. The case for and against mandatory agebased assessment of older drivers. Transport. Res., Part F 9 (5), $353-$ 362.

Levy, D.T., Vernick, J.S., Howard, K.A., 1995. Relationship between driver's license renewal policies and fatal crashes involving drivers 70 years or older. JAMA 274 (13), 1026-1030.

Li, G., Braver, E., Chen, L., 2003. Fragility versus excessive crash involvement as determinants of high death rates per vehicle-mile of travel among older drivers. Accid. Anal. Prev. 35 (2), 227-235.

Marshall, S.C., Spasoff, R., Fair, R., van Walraven, C., 2002. Restricted driver licensing for medical impairments: does it work? CMAJ 167 (7), 747-751.

Morrisey, M.A., Grabowski, D.C., 2005. State motor vehicle laws and older drivers. Health Econ. 14 (4), 407-419.

OECD, 2001. Ageing and transport: mobility needs and safety issues. Report published by an OECD Scientific Expert Group. Paris.

Royal Decree 1598/2004, de 2 de julio, por el que se modifica el Reglamento General de Conductores, aprobado por el Real Decreto 772/1997, de 30 de mayo, BOE 173 de 19 de julio de 2004, pp. 26183-26205.

The Council of the European Communities, 1991. Council Directive of 29 July 1991 on driving licenses (91/439/EEC). Official Journal L 237, 24/08/1991, pp. 0001-0024. 